\title{
ARTICULOS O
}

\section{Deterioro del fondo de comercio en las normas internacionales: estudio de una muestra de grupos cotizados españoles}

\author{
MARÍA PILAR BLASCO* \\ ALICIA COSTA** \\ ALEJANDRA MONTES***
}

* Doctora en Ciencias Económicas y Empresariales. Universidad de Zaragoza, Zaragoza, España. E-mail: mpblasco@unizar.es. ORCID: 0000-0003-4123-1799. Google Scholar: https://scholar.google.es/ citations?hl=es\&user=leqsDhgAAAA.

** Doctora en Ciencias Económicas y Empresariales. Universidad de Zaragoza, Zaragoza, España. E-mail: acosta@unizar.es. ORCID: 0000-0001-8018-7714. Google Scholar: https://scholar.google.es/citations?hl=es\&user=BzHjiLcAAAAJ.

*** Master Universitario en Auditoría de Cuentas. Universidad de Zaragoza, Zaragoza, España. E-mail: amontesataucuri@gmail.com. ORCID: 0000-0002-1515-7364. Google Scholar: https://scholar.google.com/ citations?hl=es\&authuser=1\&user=H3n3dtAAAAAJ. 


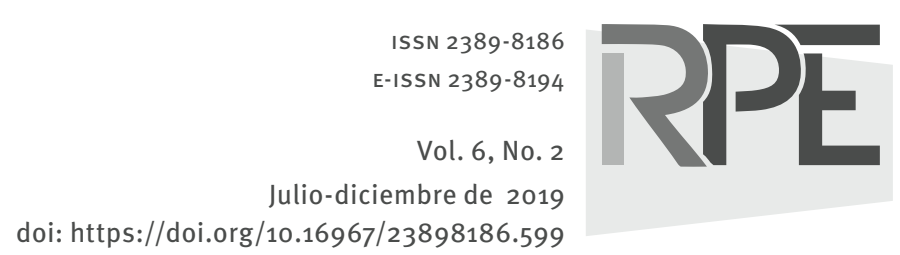

COMO CITAR ESTE ARTÍCULO

How to cite this article:

Blasco, M.P., Costa, A. y Montes, A. (2019). Deterioro del fondo de comercio en las normas internacionales: estudio de una muestra de grupos cotizados españoles. Revista Perspectiva

Empresarial, 6(2), 36-58.

Recibido: 15 de marzo de 2019

Aprobado: 08 de julio de 2019
RESUMEN El reconocimiento y valoración del fondo de comercio es una cuestión que reviste cierta complejidad, habiendo sido objeto de debate y discusión durante décadas. El objetivo de este trabajo es realizar un análisis de la normativa contable nacional e internacional respecto al fondo de comercio y contrastar su aplicación en una muestra de grupos de empresas cotizados en España, concretamente los que formaron parte del índice IBEX 35 entre 2005-2016.

Los resultados obtenidos evidencian una mejora en la calidad de la información con el paso del tiempo, si bien detectamos que las entidades son más reacias a proporcionar cierta información tal como la relacionada con los factores cualitativos que justifican el reconocimiento del fondo de comercio. El trabajo analiza también el importe del deterioro del fondo de comercio registrado en las cuentas de pérdidas y ganancias de estas compañías, observando cantidades relativamente pequeñas y en muchos casos nulas, siendo el sector financiero el que muestra -en casi todos los años- los mayores importes. Asimismo, los grupos analizados aplican en gran medida los requisitos de información establecidos por las normas en materia de valoración del fondo de comercio. Concluimos que, a pesar de la crisis económica sufrida en España durante el período objeto de estudio, los grupos registran pequeñas pérdidas de valor de este activo.

PALABRAS CLAVE fondo de comercio, deterioro de valor, normas internacionales, calidad de la información.

\section{Deterioration of the trade fund in international regulations: Study of a sample of Spanish listed groups}

ABSTRACT Recognition and valuation of the trade fund is an issue of complexity, having been the object of debate and discussion over decades. The aim of this work is to conduct an analysis of the national and international accounting regulation regarding the trade fund and to contrast its application in a sample of business groups listed in Spain, specifically those that were part of the IBEX 35 index between 2005-2016.

The results obtained evidence an improvement in information quality over time, although we detected that entities are more reluctant to providing certain information such as that related to the qualitative factors that justify the recognition of the trade fund. The work also analyses the amount of the trade fund deterioration recorded in the profit and loss accounts of these companies, observing relatively small amounts - nil in many cases-, with the financial sector showing the highest amounts in almost all the years. Furthermore, the groups analyzed apply to a great extent the information requirements established 
by the regulations in terms of trade fund valuation. We conclude that, despite the economic crisis suffered by Spain during the period under study, the groups make small losses in the value of this asset.

KEYWORDS Trade funds, value deterioration, international regulations, information quality.

\section{Deterioro do fundo de comércio nas normas internacionais: estudo de uma amostra de grupos cotizados espanhóis}

RESUMO 0 reconhecimento e valorização do fundo de comércio é uma questão que reviste certa complexidade, havendo sido objeto de debate e discussão durante décadas. 0 objetivo de este trabalho é realizar uma análise da normativa contável nacional e internacional com respeito ao fundo de comercio e contrastar sua aplicação em uma amostra de grupos de empresas cotizados na Espanha, concretamente os que formaram parte do índice IBEX 35 entre 2005-2016.

Os resultados obtidos evidenciam uma melhora na qualidade da informação com o passo do tempo, se bem detectamos que as entidades são mais relutantes a proporcionar certa informação tal como a relacionada com os fatores qualitativos que justificam o reconhecimento do fundo de comércio. 0 trabalho analisa também o importe do deterioro do fundo de comércio registrado nas contas de perdas e ganhos destas companhias, observando quantidades relativamente pequenas e em muitos casos nulas, sendo o sector financeiro o que mostra -em quase todos os anos- os maiores importes. Assim mesmo, os grupos analisados aplicam em grande medida os requisitos de informação estabelecidos pelas normas em matéria de valoração do fundo de comércio. Concluímos que, a pesar da crise económica sofrida em Espanha durante o período objeto de estudo, os grupos registram pequenas perdas de valor de este ativo.

PALAVRAS-CHAVE fundo de comércio, deterioro de valor, normas internacionais, qualidade da informação. 


\section{Introducción}

Desde 2005, los grupos cotizados españoles deben preparar sus estados financieros consolidados conforme a las normas emitidas por la International Accounting Standards Board -IASB-. Esta normativa introdujo cambios importantes como lo fue el tratamiento contable del fondo de comercio, surgido de la aplicación del método de adquisición en las operaciones de combinaciones de negocios, constituyendo este activo una parte importante del coste de las combinaciones (Forbes, 2007; Shalev, 2009; Ott and Guenther, 2010; Shalev, Zhang and Zhang, 2013).

Concretamente las entidades pasaron de amortizar el fondo de comercio referenciado a una vida útil estimada, a tener que realizar evaluaciones sobre la posible pérdida por deterioro de este activo siguiendo la nueva normativa reguladora.

Estas normas internacionales demandan información detallada tanto sobre datos básicos de la combinación de negocios como del fondo de comercio generado, además de la estimación de su posible pérdida de valor a lo largo de los años. No obstante, hoy, las exigencias del modelo utilizado para la medición del valor del fondo de comercio no están exentas de críticas al presentar un alto grado de discrecionalidad y subjetividad en su aplicación; aspecto que afectará de forma directa a la calidad de la información.

Si la información financiera presentada por las empresas es indispensable en el proceso de toma de decisiones por parte de los usuarios de la misma, esta adquiere especial importancia en el caso de los activos intangibles entre los que se encuentra el fondo de comercio por su especial naturaleza y la complejidad en su determinación.

No obstante, a pesar de su relevancia, la mayoría de los estudios realizados en distintos países del ámbito europeo revelan que la información publicada sobre estas cuestiones es incompleta y poco homogénea (Carvalho, Rodrigues and Ferreira, 2016).

El panorama descrito nos ha llevado a realizar el presente estudio cuyo objetivo principal es analizar el grado de cumplimiento por parte de los grupos cotizados españoles integrantes del IBEX 35, de la información requerida por las normas del IASB relativa al fondo de comercio y a su valoración posterior.

Para ello hemos estructurado el trabajo en varios apartados. Tras esta introducción, en los apartados segundo y tercero, realizamos una revisión sobre los cambios que han tenido lugar en la normativa contable nacional e internacional referente al tema a tratar y la literatura contable existente hasta la fecha. En el cuarto se describe la metodología empleada donde se detalla el índice no ponderado que nos permitió evaluar la calidad de la información publicada por las citadas entidades. En el quinto se analizan los principales resultados obtenidos. Por último, se muestran las conclusiones alcanzadas a través del trabajo.

\section{El tratamiento del fondo de comercio: un recorrido histórico por la normativa nacional e internacional}

La necesidad de conseguir un lenguaje común en materia contable para poder obtener información financiera comparable y fiable a escala internacional dio lugar a importantes cambios que se fueron dando de forma progresiva y finalmente desembocaron en la aplicación de las normas emitidas por el IASB dentro de Europa, afectando de forma directa a la normativa contable española por su calidad de Estado miembro de la Unión Europea (Tua, 2005; Cañibano, 2006; Cañibano y Gisbert, 2007). Si nos centramos en conocer cómo influyeron estos cambios en el tratamiento contable del fondo de comercio y su valoración posterior nos tenemos que remontar a 1970 cuando el Financial Accounting Standards Board -FASB - adoptó la norma APB 16: Business Combinations; la cual identificaba como activo al fondo de comercio y establecía su amortización en un plazo máximo de cuarenta años.

En España, en 1973, con la aprobación del primer Plan General de Contabilidad -PGC-, fue cuando aparece la primera regulación sobre el fondo de comercio. En el PGC se hablaba de un 
activo intangible generado por una transacción a título oneroso y permitía la dotación de una pérdida por deterioro correspondiente a la depreciación total o parcial contra resultado extraordinario.

Sin embargo el IASB, que también tenía en aquel momento un papel importante como organismo internacional en materia contable, no hizo mención al fondo de comercio hasta la publicación de la NIC 22 en 1983; la que, al igual que el FASB, lo reconocía cuando existía una adquisición y solo de forma residual. Esta norma establecía la amortización sistemática a lo largo de su vida útil, que como máximo estimaba era de veinte años.

No es hasta pasada década y media de la publicación del PGC del 73, que España realiza un cambio sobre su normativa contable en mayor consonancia con los modelos europeos. A partir de la Ley 19/1989 se aprueba el Real Decreto 1643/1990 donde surge una nueva versión del PGC; en el que, siguiendo la línea con lo establecido en Europa, se elimina el deterioro que se venía haciendo sobre el fondo de comercio para pasar a una amortización sistemática; allí se le asignaba una vida útil máxima de 5 años y en casos excepcionales, y debidamente justificados, se permitía hasta 10 .

Aun así, en España continuábamos en discordancia con los países del entorno. Por esto, con la intención de igualar los plazos de amortización, la Ley 37/1998 del Mercado de Valores contribuyó con este proceso al pasar a un plazo mínimo de amortización de 10 años y máximo de 20. Entretanto el FASB seguía adelantado en este campo, ya que para 2001 cambia la amortización por una prueba de deterioro de valor.

Unos años más tarde, siguiendo los pasos del FASB, el IASB sustituye la NIC 22 por la NIIF 3 sobre combinaciones de negocios; esta norma introduce un tratamiento similar al adoptado por el FASB, pues elimina la amortización del fondo de comercio y en su lugar exigía la aplicación de un test de deterioro de valor basado en la idea de unidad generadora de efectivo y en la estimación de los flujos de caja futuros generados por estas unidades.
Ambos organismos basaban su decisión en la dificultad para determinar el período en que el fondo de comercio iba a generar flujos de efectivo futuros, por ello consideraron más adecuado someterlo a una prueba para determinar su posible pérdida de valor que mantener una reducción sistemática del mismo.

En España fueron los grupos cotizados los que, a raíz de tener que aplicar las NIIF en la formulación de sus cuentas consolidadas, a partir de 1 de enero de 2005, pasaron a realizar el test de deterioro del fondo de comercio a cambio de su no amortización. Para el resto de estados, individuales y consolidados, siguiendo con el proceso de armonización contable de la información, su aplicación se produjo a través de la aprobación del PGC de 2007 (Real Decreto 1514/2007) y de las Normas para la Formulación de Cuentas Anuales Consolidadas (Real Decreto 1159/2010) respectivamente.

Por otro lado, y en el ámbito del IASB, fue en julio de 2009 cuando se vuelve a dar un nuevo giro y tras la aprobación de las NIIF para las pequeñas y medianas empresas se permite a este tipo de entidades la amortización del fondo de comercio en un plazo máximo de 10 años, a la vez que deben evaluar si existe algún indicio de deterioro de valor del citado activo.

Derivado de ello se aprobó en Europa la Directiva 2013/34/UE sobre los estados financieros individuales y consolidados, así como otros informes afines de ciertos tipos de empresas, que sustituyó a las Cuarta y Séptima Directiva que habían estado vigentes más de treinta años. Esta Directiva nace con la finalidad de simplificar las obligaciones contables de las pequeñas empresas, abriendo un nuevo proceso de armonización contable que se inició en España con la aprobación de la Ley 22/2015 de Auditoría de Cuentas desarrollada reglamentariamente a través del Real Decreto 602/2016. La citada ley reintroduce de nuevo la amortización del fondo de comercio, pero esta vez con un plazo máximo de 10 años; sin menosprecio de la necesidad de continuar analizando, al menos anualmente, si existen indicios de deterioro de su valor.

A partir de ese momento se produce algo paradójico en el país, los grupos cotizados deben aplicar las NIIF. Por lo tanto siguen sin amortizar 
el fondo de comercio a diferencia del resto de entidades que al elaborar sus cuentas de acuerdo con la normativa española, como ya hemos reseñado, deben amortizarlo en un período máximo de 10 años. Esta situación, nuevamente provoca la elaboración de información financiera con falta de comparabilidad y armonía internacional.
En la figura 1 se muestra, a modo de síntesis, cómo ha ido variando el tratamiento contable del fondo de comercio en la normativa nacional e internacional a lo largo del tiempo.

FASB

Amortización (máximo 40 años)
Test de

deterioro

\section{$1983 \quad 2004$}

IASB

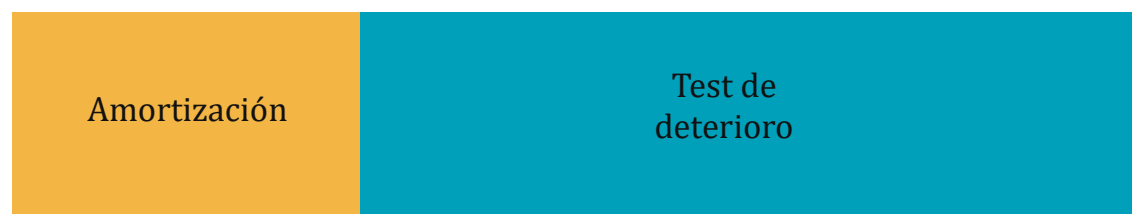

$\begin{array}{lll}1983 & 2004 & 2009\end{array}$

IASB (pymes)

\begin{tabular}{|c|c|}
\hline Amortización & $\begin{array}{c}\text { Test de } \\
\text { deterioro }\end{array}$ \\
\hline
\end{tabular}

1973

1990

2008

2016

España

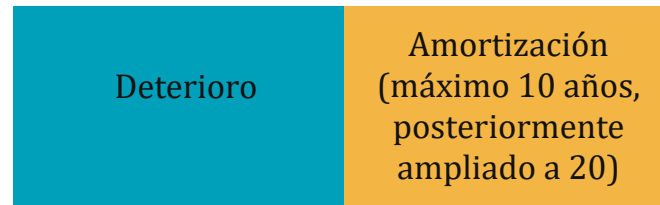

Test de

deterioro

Amortización

(10 años) y

evaluación

deterioro

1991

2005

España (grupos cotizados)

Amortización
(máximo
10 años)

Test de

deterioro

Figura 1. Evolución histórica del tratamiento contable del fondo de comercio. Fuente: elaboración propia por parte de las autoras. 


\section{Revisión de la literatura}

De todo lo visto anteriormente se desprende que la valoración posterior del fondo de comercio ha sido y sigue siendo una cuestión controvertida, por lo que cabría cuestionarse cuál es la motivación real que ha llevado a los organismos reguladores internacionales a plantear como mejor opción la de realizar un análisis por deterioro del fondo de comercio y si verdaderamente esta opción es informativamente superior a la amortización sistemática del mismo.

Calvo, Corona y Bejarano (2013) sostienen que los reguladores decidieron eliminar la amortización del fondo de comercio por considerar que el citado análisis aumenta la transparencia de los estados financieros al reflejar más fielmente la realidad económica que la amortización durante un período de tiempo arbitrario, aunque todo ello visto desde la óptica de los administradores de las entidades que puede coincidir o no con las condiciones reales.

Sin embargo numerosos estudios han demostrado que la aplicación de solo deterioro introduce un mayor grado de subjetividad derivado de la gran flexibilidad de la norma al tener que realizar una serie de hipótesis para determinar, por ejemplo, el flujo neto de efectivo que generarán las distintas unidades y la delimitación de las mismas (Wines, Dagwell and Windsor, 2007; Petersen and Plenborg, 2010; Ramanna and Watss, 2012; Avallone and Guagli, 2015; Filip, Jeanjean and Paugam, 2015; Mazzi, Liberatore and Tsalavoutas, 2016).

Esta discrecionalidad asignada al manager de la empresa ha sido duramente criticada y puede derivar en una alteración o manipulación del reconocimiento del deterioro del fondo de comercio (Watts, 2003; Beatly and Weber, 2006; Haswell and Langfield-Smith, 2008; Ramanna, 2008; Bloom, 2009; Comiskey and Mulford, 2010).

Ante esta situación que refleja evidencias de cierta discrecionalidad en la aplicación de la norma, en 2014, el European Financial Reporting
Advisory Group -EFRAG- en colaboración con el Organismo Italiano di Contabilità - OIC- y el Accounting Standards Board of Japan -ASBJplantearon ciertas alternativas al modelo actual donde se proponía entre otras el llevar a cabo un enfoque mixto entre la amortización periódica y el deterioro, realizando el test de deterioro únicamente cuando existan indicios de ello, opción que está cogiendo fuerza entre los distintos organismos nacionales e internacionales. Este es el caso del FASB que publicó la Accounting Standards Updates 2014-2 que permite amortizar el fondo de comercio en un período de 10 años, pero únicamente a las empresas no cotizadas. En el caso español, como ya hemos indicado anteriormente, ha sido la Ley de Auditoría de Cuentas la que ha introducido este tratamiento para todas las empresas y grupos no cotizados.

El IASB, en estos momentos, está trabajando en un proyecto sobre el fondo de comercio y su deterioro donde uno de los puntos a tratar es plantearse seguir solo con deterioro o bien con amortización y deterioro simultáneamente ${ }^{1}$.

No obstante, parece que la mayor o menor discrecionalidad de los gestores o directivos a la hora de reconocer un deterioro de valor del fondo de comercio está directamente relacionada con la calidad de la información proporcionada en sus memorias. Así, cuanta mayor información se disponga sobre todos los aspectos que rodean la decisión de reconocer o no una pérdida por deterioro menor probabilidad de sesgo y de comportamientos oportunistas por parte de los gestores de la entidad; y a la inversa, "la falta de cumplimiento con los requisitos de información en memoria implica dificultad para comprender las estimaciones efectuadas y limita la utilidad de la información financiera suministrada y restringe el trasvase de información interna a los mercados" (Valero, 2017, p. 235).

Como muestra tenemos el estudio realizado por Kang y Pang (2005) donde se apunta que aquellas empresas que presentan información deficitaria transmiten a sus analistas un escaso valor del fondo de comercio que llevará a su cancelación en un corto espacio de tiempo.

\footnotetext{
${ }^{1}$ El Consejo del IASB espera publicar un discussion paper en 2020.
} 
En la misma línea se encuentra el trabajo de Baboukardos y Rimmel (2014) donde se indica que la aplicación de las NIIF solo tiene un efecto positivo sobre el valor de mercado de las compañías cuando presentan altos niveles de cumplimiento de la información.

D’Alauro (2013) obtiene una asociación positiva en las empresas italianas entre su rentabilidad y el nivel informativo, en donde las empresas con bajas rentabilidades tienden a proporcionar menos datos sobre el test de deterioro del fondo de comercio. También se pueden destacar los trabajos de Botosan y Plumlee (2002), Hail (2003) y Francis, Khurana y Pereira (2005), quienes obtienen una relación inversa entre el índice informativo y el coste de capital; o el de Iatridis y Senftlechner (2014) que en su revisión de empresas australianas vuelven a resaltar la importancia que tiene para los usuarios presentar con detalle la información para poder obtener fácil acceso al dinero y al mercado de capitales.

Lazar y Velte (2018), quienes trabajan con entidades alemanas en los ejercicios de 2010 a 2015, obtuvieron que la rentabilidad de la empresa y las pérdidas por deterioro del fondo de comercio tienen una relación positiva con la calidad de la información publicada.

No obstante, a pesar de estas evidencias sobre la eficacia de la revelación de información y de la importancia de comprender los procesos de concentración empresarial al tener en cuenta la complejidad y subjetividad implícita que rodea la determinación del fondo de comercio, no parece que haya sido asumido por los responsables de aplicar la norma y así lo demuestran múltiples estudios llevados a cabo en distintos países de la Unión Europea centrados en uno o varios años y en uno o varios países.

Así pues, Feteke, Matis y Lukacs (2008) detectan que el nivel medio de divulgación de todo lo relacionado con este activo intangible por parte de las empresas cotizadas en Hungría es del $62 \%$. Mientras que Carlin y Finch (2010) realizan el estudio en compañías australianas, Ballas y Tzovas (2010) y Tsalavoutas y Dionysiou (2014) lo efectúan con sociedades ubicadas en Grecia. Las conclusiones alcanzadas en todos ellos es, en mayor o menor medida, un elevado índice de incumplimiento de la normativa.

Lo mismo ocurre en la investigación realizada por Devalle y Rizatto (2012) donde analizan la información publicada para el ejercicio 2010 por entidades situadas en Italia, Francia, Alemania y España, en donde solo el $27 \%$ de la muestra informa correctamente sobre los requerimientos exigidos sobre el fondo de comercio.

Estos estudios utilizan datos de un solo año, y en la mayor parte de los casos coincidente con los primeros años de aplicación de la norma. Existen otros trabajos que utilizan un período más extenso de tiempo como los llevados a cabo por Guthrie y Pang (2013) en empresas australianas entre 2005-2010; Hartwing (2015) en sociedades cotizadas de Suecia y Holanda desde 2005 hasta 2008; Biancone (2012) e Izzo, Luciani y Sartori (2013) que analizan empresas italianas (de 2007 a 2009 los primeros, y los segundos ampliando el plazo hasta 2011) mostrando que, a pesar de haber transcurrido varios años de la aplicación de la norma y de haber mejorado con el paso del tiempo, sigue siendo muy incompleta en algunos aspectos; destacan estos últimos autores, en especial aquellos puntos relacionados con la determinación de los flujos de caja esperados y las tasas de descuento y crecimiento.

En la misma línea está el trabajo de Camodeca, Almici y Bernardi (2013) efectuado a compañías de Reino Unido, para el período 2007 a 2011, evidenciando una ausencia de divulgación especialmente los puntos relacionados con el valor recuperable del activo (acentuada en los años posteriores a la crisis financiera, es decir, de 2009 a 2011).

Esta situación, fruto de la importancia de proporcionar información completa y relevante a los usuarios de los estados financieros y más concretamente sobre el fondo de comercio elemento difícil de comprender y valorar-, nos ha llevado a realizar este trabajo. Siguiendo la línea de Calvo (2011), en la que se analizaban las empresas del IBEX 35 para los años 2005 a 2009, hemos ampliado el período objeto de estudio a doce años; el estudio se cubrió desde el ejercicio 2005 (fecha de implantación de las NIIF en España para los grupos cotizados) hasta 2016 (último año del que disponíamos datos). 


\section{Metodología}

Para alcanzar el objetivo de este trabajo hemos examinado en una muestra de grupos cotizados españoles, concretamente los que formaban parte del IBEX 35 a julio de 2017, la información contenida en las notas a los estados financieros consolidados en el período comprendido entre 2005 (primer año de aplicación de las normas internacionales) y 2016. Se han excluido todos los casos en los que no se disponía de información consolidada, así como los que no tenían fondo de comercio, lo que nos ha llevado a trabajar con un total de 324 observaciones.

La información de cada uno de los grupos, la obtuvimos del registro oficial de cuentas anuales incluido en la página Web de la Comisión Nacional del Mercado de Valores. Elaboramos un índice formado por 14 ítems que recogen la información exigida por las distintas normativas y que a los efectos de nuestro trabajo agrupamos en las cuatro secciones que se detallan a continuación. Conviene matizar que, en función de sus políticas contables, no todos los grupos deben revelar información sobre las cuatro secciones.

Sección A: información general sobre la combinación de negocios y el fondo de comercio.

Sección B: información sobre el importe recuperable de la unidad (o grupo de unidades) cuando se utiliza el valor de uso.

Sección C: información sobre el importe recuperable de la unidad (o grupo de unidades) cuando se utiliza el valor razonable menos coste de venta.

Sección D: información, en su caso, sobre el reconocimiento o reversión de pérdidas por deterioro de valor del fondo de comercio.

Teniendo en cuenta que este estudio pretende medir el nivel informativo, sin atender a necesidades particulares o preferencias definidas por tipos de usuarios concretos, hemos optado por utilizar un índice no ponderado ${ }^{2}$.
Esto significa que a todos los ítems se le otorga el mismo valor y el índice se obtiene por simple agregación de la puntuación asignada a cada uno de ellos; es decir se parte de la hipótesis de que todos los aspectos analizados tienen la misma importancia.

La puntuación que se asigna es de 1 punto cuando la información es completa, de 0,5 cuando falta algún dato y de 0 cuando no informa.

Todas las entidades deben informar como mínimo las cuestiones de la sección A, a partir de ahí la valoración dependía de la base utilizada para calcular el valor recuperable y de si ha reconocido o no pérdida por deterioro de valor del fondo de comercio.

Por ello hemos dividido las distintas opciones que nos podemos encontrar en cuatro bloques, indicando la nota máxima que puede obtenerse en cada uno.

Bloque 1: el valor en uso es la base para calcular el importe recuperable y no ha reconocido pérdida por deterioro del valor del fondo de comercio. Nota máxima, 8 puntos.

Bloque 2: el valor en uso es la base para calcular el importe recuperable y ha reconocido pérdida por deterioro del valor del fondo de comercio. Nota máxima, 12 puntos.

Bloque 3: el valor razonable menos coste de venta es la base para calcular el importe recuperable y no ha reconocido pérdida por deterioro del valor del fondo de comercio. Nota máxima, 5 puntos.

Bloque 4: el valor razonable menos coste de venta es la base para calcular el importe recuperable y ha reconocido pérdida por deterioro del valor del fondo de comercio. Nota máxima, 9 puntos.

2 Otros estudios que utilizan índices no ponderados son los de Paananen (2008), Chen y Gu (2011), Biancone (2012), Devalle y Rizzato (2012), D’Alauro (2013), Bepari, Rahman y Mollik (2014) entre otros. 
Tabla 1. Puntuación máxima catalogada en bloques

\begin{tabular}{lccccc}
\hline \hline & Nota máxima & Bloque 1 & Bloque 2 & Bloque 3 & Bloque 4 \\
\hline Sección A & 4 & $\boldsymbol{J}$ & $\boldsymbol{J}$ & $\boldsymbol{J}$ \\
Sección B & 4 & $\boldsymbol{J}$ & & & \\
Sección C & 1 & & $\boldsymbol{J}$ & $\boldsymbol{J}$ \\
Sección D & 4 & $\mathbf{8}$ & $\mathbf{1 2}$ & $\mathbf{5}$ & $\mathbf{9}$ \\
& Total & & &
\end{tabular}

Fuente: elaboración propia por parte de las autoras.

\section{Resultados obtenidos}

Una vez revisadas las notas a los estados financieros que divulgaron cada grupo cotizado, configuramos las tablas 2 y 3 ; en estas se muestra el porcentaje de información revelada en cada uno de los sectores en los que se localizan las distintas entidades y en cada uno de los años analizados, considerando la puntuación máxima que podría alcanzarse en función de las características de base del grupo.

Observamos cómo, en todos los sectores, el grado medio de cumplimiento de los requisitos establecidos por las normas internacionales experimenta -como era de esperar- una mejora con el paso de los años; a excepción del sector del petróleo y energía que disminuye más de 10 puntos porcentuales a partir del ejercicio 2012. Esta situación se debe a la incorporación del grupo Enagás, el cual no proporciona información suficiente sobre la base de cálculo del valor recuperable de la unidad generadora de efectivo y ello hace que tenga una puntuación muy baja.

Comparativamente es el sector financiero el que ha experimentado una mejora sustancial de sus cifras, presentando en los primeros años una información muy débil que llega a alcanzar unos valores del $81 \%$ en 2016. Dentro de este sector hay grupos que experimentan una variación hasta de un $80 \%$ entre sus valores mínimos y máximos, mejoras que se dan fundamentalmente a partir de los años de fuerte crisis en el sector (esto es, desde 2008).

En los grupos analizados, a pesar de estar sujetos a altos controles de supervisión al formar parte del IBEX 35, sorprende no encontrar alguno que presente una información completa; pues en todos ellos hay algún dato que no es facilitado. 
Tabla 2. Información proporcionada por sector y año analizado

\begin{tabular}{|c|c|c|c|c|c|c|c|c|c|c|c|c|}
\hline Sector & 2005 & 2006 & 2007 & 2008 & 2009 & 2010 & 2011 & 2012 & 2013 & 2014 & 2015 & 2016 \\
\hline $\begin{array}{l}\text { Bienes de } \\
\text { consumo }\end{array}$ & $61 \%$ & $72 \%$ & $72 \%$ & $72 \%$ & $72 \%$ & $72 \%$ & $82 \%$ & $84 \%$ & $84 \%$ & $84 \%$ & $82 \%$ & $83 \%$ \\
\hline $\begin{array}{l}\text { Materiales } \\
\text { básicos e indutria }\end{array}$ & $70 \%$ & $72 \%$ & $73 \%$ & $75 \%$ & $75 \%$ & $76 \%$ & $78 \%$ & $78 \%$ & $86 \%$ & $85 \%$ & $84 \%$ & $84 \%$ \\
\hline $\begin{array}{l}\text { Petróleo y } \\
\text { energía }\end{array}$ & $62 \%$ & $62 \%$ & $62 \%$ & $73 \%$ & $73 \%$ & $73 \%$ & $83 \%$ & $71 \%$ & $70 \%$ & $70 \%$ & $70 \%$ & $70 \%$ \\
\hline $\begin{array}{l}\text { Servicios de } \\
\text { consumo }\end{array}$ & $76 \%$ & $76 \%$ & $76 \%$ & $76 \%$ & $81 \%$ & $81 \%$ & $80 \%$ & $79 \%$ & $80 \%$ & $81 \%$ & $81 \%$ & $81 \%$ \\
\hline $\begin{array}{l}\text { Servicios } \\
\text { financieros e } \\
\text { inmobiliarios }\end{array}$ & $37 \%$ & $52 \%$ & $42 \%$ & $66 \%$ & $73 \%$ & $68 \%$ & $77 \%$ & $77 \%$ & $77 \%$ & $78 \%$ & $81 \%$ & $81 \%$ \\
\hline $\begin{array}{l}\text { Tecnología y } \\
\text { telecomunicaciones }\end{array}$ & $78 \%$ & $65 \%$ & $77 \%$ & $80 \%$ & $82 \%$ & $83 \%$ & $83 \%$ & $85 \%$ & $81 \%$ & $83 \%$ & $83 \%$ & $83 \%$ \\
\hline
\end{tabular}

Fuente: elaboración propia por parte de las autoras.

Tabla 3. Análisis descriptivo por sectores

\begin{tabular}{|c|c|c|c|c|}
\hline Sector & Media & Mínimo & Máximo & Desviación típica \\
\hline Bienes de consumo & $77 \%$ & $61 \%$ & $84 \%$ & 0,08 \\
\hline Materiales básicos e industria & $78 \%$ & $70 \%$ & $86 \%$ & 0,05 \\
\hline Petróleo y energía & $70 \%$ & $62 \%$ & $83 \%$ & 0,06 \\
\hline Servicios de consumo & $79 \%$ & $76 \%$ & $81 \%$ & 0,02 \\
\hline Servicios financieros e inmobiliarios & $68 \%$ & $37 \%$ & $81 \%$ & 0,15 \\
\hline Tecnología y telecomunicaciones & $80 \%$ & $65 \%$ & $85 \%$ & 0,05 \\
\hline
\end{tabular}

Fuente: elaboración propia por parte de las autoras.

Por ello con el objeto de ver dónde se localizan las mayores carencias de información hemos elaborado la tabla 4. Allí se puede ver en términos totales cuál es la información que más cuesta encontrar en las notas a los estados consolidados de todos los grupos y cuál se muestra sin mayor dificultad. 
Tabla 4. Información detallada por sector y sección

\begin{tabular}{|c|c|c|c|c|c|c|c|}
\hline & $\begin{array}{l}\text { Bienes } \\
\text { consumo }\end{array}$ & $\begin{array}{c}\text { Material } \\
\text { básico }\end{array}$ & $\begin{array}{l}\text { Petróleo y } \\
\text { energía }\end{array}$ & $\begin{array}{l}\text { Servicios } \\
\text { consumo }\end{array}$ & $\begin{array}{c}\text { Servicios } \\
\text { financieros }\end{array}$ & Tecnología & Media \\
\hline Sección A & $74 \%$ & $69 \%$ & $68 \%$ & $37 \%$ & $63 \%$ & $69 \%$ & $63 \%$ \\
\hline $\begin{array}{l}\text { 1. ¿Datos básicos sobre la } \\
\text { combinación de negocios? }\end{array}$ & $100 \%$ & $92 \%$ & $100 \%$ & $57 \%$ & $78 \%$ & $92 \%$ & $86 \%$ \\
\hline $\begin{array}{l}\text { 2. ¿Describe los factores } \\
\text { que constituyen el fondo de } \\
\text { comercio? }\end{array}$ & $0 \%$ & $0 \%$ & $0 \%$ & $0 \%$ & $0 \%$ & $0 \%$ & $0 \%$ \\
\hline $\begin{array}{l}\text { 3. ¿El importe en libros del fondo } \\
\text { de comercio distribuido a la/s } \\
\text { UGE? }\end{array}$ & $100 \%$ & $98 \%$ & $92 \%$ & $35 \%$ & $91 \%$ & $83 \%$ & $83 \%$ \\
\hline $\begin{array}{l}\text { 4. ¿La base sobre la que ha } \\
\text { determinado el importe } \\
\text { recuperable de la/s UGE? }\end{array}$ & $97 \%$ & $85 \%$ & $79 \%$ & $57 \%$ & $84 \%$ & $100 \%$ & $84 \%$ \\
\hline Sección B & $81 \%$ & $93 \%$ & $72 \%$ & $97 \%$ & $62 \%$ & $91 \%$ & $83 \%$ \\
\hline $\begin{array}{l}\text { 5. ¿Hipótesis clave sobre las } \\
\text { que la dirección ha basado sus } \\
\text { proyecciones? }\end{array}$ & $75 \%$ & $77 \%$ & $45 \%$ & $91 \%$ & $64 \%$ & $63 \%$ & $69 \%$ \\
\hline $\begin{array}{l}\text { 6. ¿Período de proyección de los } \\
\text { flujos de efectivo? }\end{array}$ & $100 \%$ & $95 \%$ & $81 \%$ & $98 \%$ & $64 \%$ & $100 \%$ & $90 \%$ \\
\hline $\begin{array}{l}\text { 7. ¿Tasa de crecimiento para } \\
\text { extrapolar las proyecciones de } \\
\text { flujos de efectivo? }\end{array}$ & $75 \%$ & $100 \%$ & $81 \%$ & $100 \%$ & $61 \%$ & $100 \%$ & $86 \%$ \\
\hline $\begin{array}{l}\text { 8. ¿El tipo o tipos de descuento } \\
\text { aplicados a las proyecciones? }\end{array}$ & $75 \%$ & $100 \%$ & $81 \%$ & $100 \%$ & $61 \%$ & $100 \%$ & $86 \%$ \\
\hline
\end{tabular}

\section{Sección C}

9. ¿Metodología empleada para

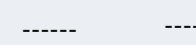

determinar el valor razonable?

10. ¿Hipótesis clave para

$67 \%$

$33 \%$

$50 \%$

$100 \%$

$100 \%$

determinar el valor

razonable? 


\begin{tabular}{|c|c|c|c|c|c|c|c|}
\hline & $\begin{array}{c}\text { Bienes } \\
\text { consumo }\end{array}$ & $\begin{array}{l}\text { Material } \\
\text { básico }\end{array}$ & $\begin{array}{l}\text { Petróleo y } \\
\text { energía }\end{array}$ & $\begin{array}{l}\text { Servicios } \\
\text { consumo }\end{array}$ & $\begin{array}{c}\text { Servicios } \\
\text { financieros }\end{array}$ & Tecnología & Media \\
\hline Sección D & $75 \%$ & $95 \%$ & $100 \%$ & $60 \%$ & $86 \%$ & $100 \%$ & $86 \%$ \\
\hline $\begin{array}{l}\text { 11. ¿Eventos que han llevado al } \\
\text { reconocimiento o reversión de la } \\
\text { pérdida por deterioro? }\end{array}$ & $0 \%$ & $81 \%$ & $100 \%$ & $0 \%$ & $94 \%$ & $100 \%$ & $63 \%$ \\
\hline $\begin{array}{l}\text { 12. ¿Pérdidas por deterioro de } \\
\text { valor acumuladas al principio del } \\
\text { ejercicio? }\end{array}$ & $100 \%$ & $100 \%$ & $100 \%$ & $80 \%$ & $87 \%$ & $100 \%$ & $94 \%$ \\
\hline $\begin{array}{l}\text { 13. ¿Pérdidas por deterioro de } \\
\text { valor reconocidas durante el } \\
\text { ejercicio? }\end{array}$ & $100 \%$ & $100 \%$ & $100 \%$ & $80 \%$ & $100 \%$ & $100 \%$ & $97 \%$ \\
\hline $\begin{array}{l}\text { 14. ¿Pérdidas por deterioro de } \\
\text { valor acumuladas al final del } \\
\text { ejercicio? }\end{array}$ & $100 \%$ & $100 \%$ & $100 \%$ & $80 \%$ & $65 \%$ & $100 \%$ & $91 \%$ \\
\hline
\end{tabular}

Fuente: elaboración propia por parte de las autoras.

La sección A, en la que se recoge la información general que deben presentar todas las entidades y relativa a la combinación de negocios y el fondo de comercio, presenta carencias importantes. Observamos una ausencia total de datos sobre los factores cualitativos que justifican el reconocimiento del fondo de comercio, resultado alcanzado igualmente en el trabajo de Calvo (2011).

En el resto de apartados de esta sección, la media es elevada con valores superiores al $80 \%$; aunque existe cierta disparidad entre los sectores, concretamente el de servicios de consumo refleja unos valores inferiores al resto, siendo muy deficiente la información proporcionada sobre el importe en libros del fondo de comercio distribuido entre las unidades generadoras de efectivo.

Sin embargo y curiosamente es este sector el que mayor información proporciona sobre el importe recuperable de estas unidades tanto si se utiliza el valor en uso como el valor razonable, la media es prácticamente del $100 \%$. Estos datos están reflejados en las secciones B y C respectivamente.

En relación al importe recuperable, la mayoría de los grupos utilizan como base para su cálculo el valor en uso; tan solo 6 de las 33 entidades analizadas $^{3}$ emplean el valor razonable menos coste de venta, no existiendo un factor común entre ellas al pertenecer a sectores diferentes tal y como puede verse en la figura 2. Resultados similares obtienen Petesern y Plenborg (2010), Camodeca, Almici y Bernardi (2013) e Izzo, Luciani y Sartori (2013).

3 Estas compañías son: Banco Santander; Inmobiliaria Colonial; Meliá Hotels; Repsol; Acciona y Viscofan. 


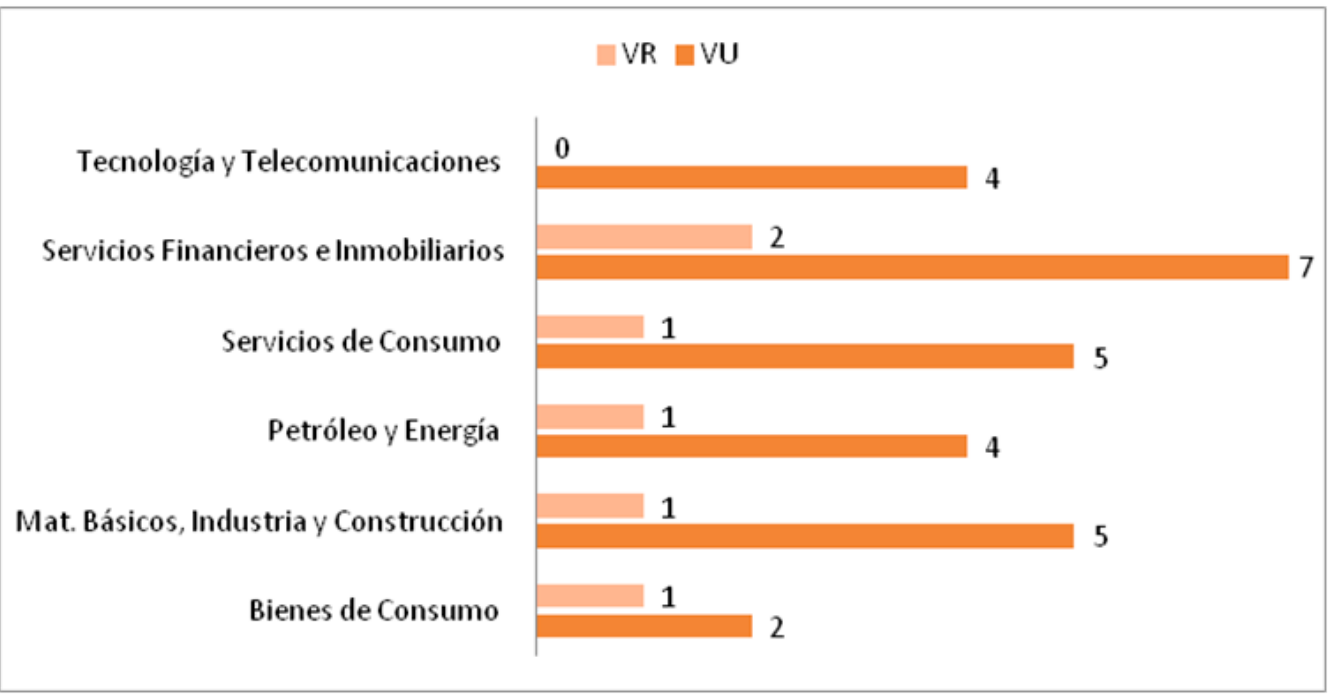

Figura 2. Base utilizada para el cálculo del importe recuperable por sectores. Fuente: elaboración propia por parte de las autoras.

En los grupos que utilizan el valor razonable y no disponen de un precio de mercado observable la información presentada es prácticamente nula hasta el ejercicio 2011 en algunos casos y en otros hasta 2013, esto hace que sus medias no sean muy altas.

En lo que respecta a la información requerida sobre la aplicación del valor en uso 4 la respuesta media de la sección B es del $83 \%$, siendo el sector financiero el que menor información presenta; $y$, por el contrario, como lo hemos comentado anteriormente, es el sector servicios de consumo el que muestra un índice de respuesta cercano al 100 $\%$. Dentro de esta sección las hipótesis clave sobre las que la dirección ha basado sus proyecciones de flujos de efectivo no son presentadas con la suficiente claridad.

$\mathrm{Al}$ ser el valor en uso el tomado mayoritariamente como base para el cálculo del importe recuperable de la(s) unidad(es) generadora(s) de efectivo de la(s) que forma parte el fondo de comercio, vamos a evaluar las características de los parámetros aplicados para determinarlo.
En concreto, como establece la NIC 36 (art. 134, (d), (iii) y (iv)), hemos revisado el período sobre el que la dirección ha proyectado los flujos de efectivo y la tasa de crecimiento empleada para extrapolar las proyecciones de estos flujos y el tipo de descuento aplicado. El detalle de estos aspectos reportados queda recogido en la tabla 5 .

${ }^{4}$ Hemos excluido del estudio al grupo Enagás al no disponer de datos. 
Tabla 5. Parámetros utilizados para calcular el valor en uso

\begin{tabular}{|c|c|c|c|c|c|}
\hline \multirow{2}{*}{ Grupo } & \multirow{2}{*}{$\begin{array}{l}\text { Período de } \\
\text { proyección }\end{array}$} & \multicolumn{2}{|c|}{ Tasa de crecimiento } & \multicolumn{2}{|c|}{ Tipo de descuento } \\
\hline & & Mínimo & Máximo & Mínimo & Máximo \\
\hline \multicolumn{6}{|l|}{ Bienes de consumo } \\
\hline GRIFOLS-A & 5 & - & $2 \%$ & $9,10 \%$ & $10,80 \%$ \\
\hline INDITEX & 3 a 5 & - & $2 \%$ & & $7,20 \%$ \\
\hline \multicolumn{6}{|l|}{ Materiales básicos } \\
\hline ACERINOX & 5 & - & $2,50 \%$ & - & $11,70 \%$ \\
\hline ACS & 5 & - & $2,50 \%$ & - & $9,47 \%$ \\
\hline FERROVIAL & 5 & - & $2 \%$ & $7,60 \%$ & $8,10 \%$ \\
\hline GAMESA & 5 & - & $1 \%$ & - & $8,50 \%$ \\
\hline TECNICAS REUNIDAS & 5 & - & $1 \%$ & - & $9,75 \%$ \\
\hline \multicolumn{6}{|l|}{ Petróleo y energía } \\
\hline ENAGAS & - & - & - & - & - \\
\hline ENDESA & 5 & $1,40 \%$ & $1,80 \%$ & $5,90 \%$ & $8,10 \%$ \\
\hline GAS NATURAL SDG & 5 & $0 \%$ & $1 \%$ & $3 \%$ & $13 \%$ \\
\hline IBERDROLA & 10 & - & $2,30 \%$ & $4,90 \%$ & $10,64 \%$ \\
\hline \multicolumn{6}{|l|}{ Servicios de consumo } \\
\hline ABERTIS & 5 a 36 & - & $2 \%$ & $5,25 \%$ & $12,62 \%$ \\
\hline AENA & 5 & $1,50 \%$ & $2 \%$ & $5,23 \%$ & $5,33 \%$ \\
\hline DIA & 5 & - & $3 \%$ & $6,88 \%$ & $7,49 \%$ \\
\hline INT. AIRLINES GROUP & 5 & $2,20 \%$ & $2,50 \%$ & $10 \%$ & $12,50 \%$ \\
\hline MEDIASET ESPAÑA & 4 & - & $2 \%$ & $9 \%$ & $11 \%$ \\
\hline \multicolumn{6}{|l|}{ Servicios financieros } \\
\hline BANCO SABADELL & 5 & - & $0 \%$ & - & $9,5 \%$ \\
\hline BANKIA & 5 & $1 \%$ & $2 \%$ & - & $10,00 \%$ \\
\hline BANKINTER & 5 & - & $2 \%$ & - & $10 \%$ \\
\hline BBVA & 5 & - & $4 \%$ & - & $9,80 \%$ \\
\hline CAIXABANK & 3 & - & $2 \%$ & - & $9,30 \%$ \\
\hline MAPFRE & 5 & - & $1,50 \%$ & - & $7,50 \%$ \\
\hline MERLIN PROPERTIES & 10 & $1,90 \%$ & $2,90 \%$ & $7 \%$ & $7,50 \%$ \\
\hline \multicolumn{6}{|l|}{ Tecnología } \\
\hline AMADEUS IT GROUP & 5 & $-1 \%$ & $2,50 \%$ & - & $7,80 \%$ \\
\hline CELLNEX TELECOM & 5 & $0 \%$ & - & - & $8,16 \%$ \\
\hline INDRA SISTEMAS BR-A & 5 & $1 \%$ & $4,93 \%$ & $11,93 \%$ & $12,30 \%$ \\
\hline TELEFONICA & 5 & - & $0,70 \%$ & - & $6,10 \%$ \\
\hline
\end{tabular}


Vemos como la mayoría de estos utilizan un período de proyección de 5 años y únicamente 3 superan este límite, planteamiento un tanto arriesgado porque resulta difícil predecir los flujos de efectivo de forma precisa en plazos tan dilatados de tiempo.

En relación a la tasa de crecimiento la NIC 36 (art. 33) indica que debe ser constante o decreciente, salvo que se pueda justificar el uso de una tasa creciente en el tiempo. La tasa más elevada la aplica Indra con un valor máximo de $4,93 \%$, en el resto de grupos oscila entre $-1 \%$ y $4 \%$.

Al igual que en el trabajo llevado a cabo por Pietro (2012), el cálculo más utilizado para la tasa de descuento ha sido el de coste medio ponderado del capital -CMPC-; las hipótesis empleadas para calcular el tipo libre de riesgo y la prima de mercado son consistentes con el horizonte temporal de los flujos a descontar y con las expectativas de inflación. Asimismo, se aplican tasas diferentes en función del perfil de riesgo de la unidad.
Un poco más de la mitad de los grupos indican una única tasa de descuento y el resto establecen un rango determinado, que en el caso de Gas Natural alcanza los 10 puntos porcentuales.

Por último, en la sección D se muestra la información relacionada con el reconocimiento de las pérdidas de valor del fondo de comercio. Concretamente los eventos que han llevado a su contabilización y las pérdidas acumuladas al principio, durante y final del ejercicio.

Como se observa en las tablas 6 y 7 los grupos que han reconocido deterioro han ido creciendo con el paso de los años, pasando de ser 2 en el ejercicio $2005(8,7 \%)$ a 12 en el $2012(41,38 \%)$; aunque en los ejercicios siguientes se reducen a 6 y 7, situación por otro lado lógica al encontrarnos en un período de recuperación en nuestro país. No obstante, aun en los años en que mayor número de empresas registran deterioro este no es elevado al considerar la situación de crisis económica vivida recientemente en España; aunque entendemos que esto puede explicarse por las características de la muestra utilizada.

Tabla 6. Número de entidades que reconocen deterioro cada año

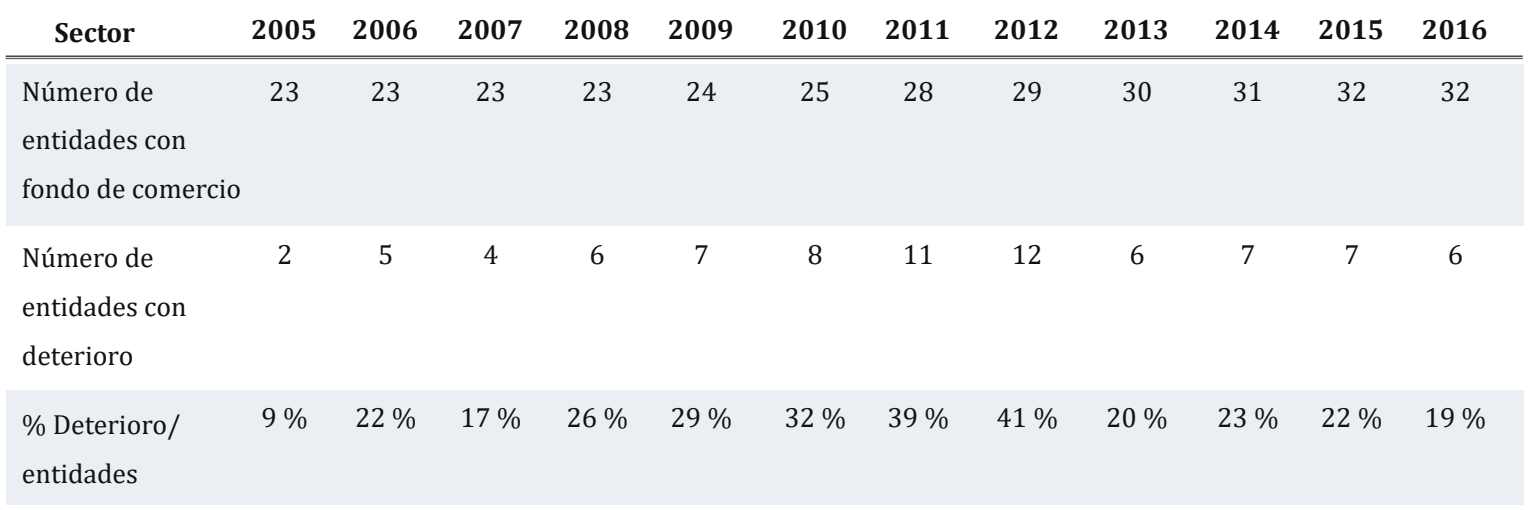

Fuente: elaboración propia por parte de las autoras. 
Tabla 7. Número de entidades que reconocen deterioro cada año por sectores

\begin{tabular}{|c|c|c|c|c|c|c|c|c|c|c|c|c|}
\hline Sector & 2005 & 2006 & 2007 & 2008 & 2009 & 2010 & 2011 & 2012 & 2013 & 2014 & 2015 & 2016 \\
\hline $\begin{array}{l}\text { Bienes de } \\
\text { consumo }\end{array}$ & & & & & & & 1 & & & & 1 & \\
\hline Materiales básicos & 1 & 1 & 1 & 1 & 1 & 1 & 1 & 1 & 2 & 1 & 2 & 2 \\
\hline Petróleo y energía & & & & & 1 & 1 & 1 & 1 & & & & \\
\hline $\begin{array}{l}\text { Servicios de } \\
\text { consumo }\end{array}$ & & & & & & & 1 & 2 & 1 & 1 & & 1 \\
\hline $\begin{array}{l}\text { Servicios } \\
\text { financieros }\end{array}$ & 1 & 4 & 3 & 4 & 4 & 5 & 6 & 6 & 2 & 4 & 3 & 2 \\
\hline Tecnología & & & & 1 & 1 & 1 & 1 & 2 & 1 & 1 & 1 & 1 \\
\hline
\end{tabular}

Fuente: elaboración propia por parte de las autoras.

El sector financiero es el que presenta un mayor número de grupos que deterioran su fondo de comercio, alcanzando su cuota más alta en los ejercicios 2011 y 2012 en los que 6 de los 8 grupos del sector que disponían de este activo en sus balances han contabilizado pérdidas por deterioro.

La información mostrada sobre este aspecto puede considerarse satisfactoria al presentar en diversas cuestiones y en varios sectores una información completa (tabla 4). Vuelve a ser el sector de servicios de consumo el que muestra importes inferiores al resto; aunque, como se puede apreciar en la tabla 7 , solo uno o dos de los grupos de este sector recogen deterioro y no en todos los años.

Si examinamos en los grupos que contabilizan deterioro el porcentaje que este representa sobre la cuantía del fondo de comercio reconocido en el balance en cada ejercicio económico, importe que ha sido corregido para no considerar el deterioro de ese mismo año (tabla 8), vemos en términos generales valores por debajo del $5 \%$; lo que hubiera sido el gasto contabilizado en caso de haber seguido el criterio de amortizar tal como hacen en este momento los grupos españoles que no cotizan en el mercado de valores. 
Tabla 8. Porcentaje de deterioro del fondo de comercio por sectores y año

\begin{tabular}{|c|c|c|c|c|c|c|c|c|c|c|c|c|c|}
\hline Sector & 2005 & 2006 & 2007 & 2008 & 2009 & 2010 & 2011 & 2012 & 2013 & 2014 & 2015 & 2016 & Media \\
\hline $\begin{array}{l}\text { Bienes de } \\
\text { consumo }\end{array}$ & & & & & & & 0,68 & & & & 0,07 & & 0,38 \\
\hline $\begin{array}{l}\text { Materiales } \\
\text { básicos }\end{array}$ & 0,25 & 0,00 & 0,23 & 0,42 & 0,02 & 0,01 & 0,01 & 0,11 & 1,04 & 0,66 & 1,49 & 0,65 & 0,41 \\
\hline $\begin{array}{l}\text { Petróleo y } \\
\text { energía }\end{array}$ & & & & & 2,83 & 3,95 & 0,30 & 0,52 & & & & & 1,90 \\
\hline $\begin{array}{l}\text { Servicios de } \\
\text { consumo }\end{array}$ & & & & & & & 0,62 & 41,79 & 0,01 & 0,01 & & 0,05 & 8,49 \\
\hline $\begin{array}{l}\text { Servicios } \\
\text { financieros }\end{array}$ & 2,91 & 1,83 & 6,32 & 18,56 & 8,16 & 10,54 & 9,91 & 23,72 & 7,18 & 28,45 & 2,64 & 2,15 & 10,20 \\
\hline Tecnología & & & & 0,23 & 0,33 & 0,12 & 0,54 & 1,08 & 2,28 & 5,77 & 22,99 & 23,05 & 6,26 \\
\hline
\end{tabular}

Fuente: elaboración propia por parte de las autoras.

Solo hay dos grupos que reconocen pérdidas todos los años, ACS y Mapfre, siendo los valores promedio del deterioro de 0,22 \% y 5,28 \% respectivamente.

El sector financiero es el que registra los mayores porcentajes de pérdidas en casi todo el período, alcanzando cifras de hasta un $23,72 \%$ y $28,45 \%$ debido principalmente a que Bankia (en 2012) e Inmobiliaria Colonial (en 2014) han deteriorado la totalidad de su fondo de comercio. Asimismo, el grupo International Consolidated Airlines del sector de servicios de consumo también reconoce, en 2012, pérdidas cercanas al $100 \%$.

En estas compañías vemos claramente reflejado el efecto de la crisis económica sufrida en nuestro país en los pasados años, en especial en las del sector financiero fuertemente castigado por esta situación de recesión.

\section{Conclusiones}

Este trabajo analiza la información divulgada por los grupos integrantes del IBEX 35 en relación al fondo de comercio y la estimación de su deterioro. La investigación está motivada por los movimientos habidos en las últimas décadas en la normativa contable nacional e internacional sobre el tratamiento contable de este intangible y la información a presentar sobre el mismo.

Los grupos cotizados en nuestro país sufrieron en el ejercicio 2005 un cambio normativo muy importante, pues se paso a tener la obligación de aplicar la normativa internacional para elaborar sus cuentas anuales consolidadas. A pesar de tratarse de entidades de gran volumen de negocio, y que por lo tanto cuentan con personal cualificado en sus distintos departamentos de control y gestión, tuvieron que dedicar importantes recursos para adaptarse a la nueva situación y ello inevitablemente se vio reflejado en la información reportada en los estados financieros presentados en los primeros años de implantación de las NIIF.

Entre los diversos cambios que tuvieron que abordar hemos centrado nuestra investigación en uno de ellos, en la valoración otorgada al fondo de comercio surgido de una combinación de negocios. Este activo que venía siendo amortizado a lo largo de su vida útil pasó a no amortizarse tal como venía regulado por la NIIF 3, debiendo aplicarse solo un test de deterioro como mínimo anualmente para poder determinar y reconocer 
las posibles pérdidas de valor sufridas por el citado activo.

A este nuevo tratamiento le rodea cierto grado de subjetividad por la discrecionalidad que se le otorga a los directivos en las estimaciones a llevar a cabo con el objeto de poder determinar si realmente este activo sigue valiendo el importe pagado por él o, por el contrario, está reduciendo su valor.

Esta discrecionalidad debe ser compensada con la información que deben proporcionar las empresas al respecto, encontrándose una relación directa entre ambas; es decir que cuanta mayor información se tenga sobre el proceso seguido en la aplicación del test de deterioro menor probabilidad de manipulación de los datos existirá.

En esto se ha centrado nuestro trabajo, analizando si una muestra de los grupos cotizados que forman parte del IBEX 35 ha cumplido con las exigencias establecidas por las NIIF desde el año de su implantación hasta el día de hoy.

Para ello se ha elaborado un índice no ponderado con 14 ítems donde se recogen tanto datos generales sobre la combinación de negocios que ha generado el fondo de comercio como del propio intangible; de igual manera observando el valor recuperable sobre el que se ha centrado el cálculo del posible deterioro, valor en uso o valor razonable neto y los datos sobre la pérdida de valor experimentada por sí mismo.

Los resultados extraídos muestran en los distintos sectores de actividad en los que operan las empresas unos niveles -en términos de medias- iguales o superiores al $68 \%$ de revelación de información, alcanzando sus cuotas máximas una vez transcurridos varios años de aplicación de las NIIF.

El sector financiero es el que muestra una mayor variación con el paso de los años, pasando de divulgar un $37 \%$ de los datos exigidos en el ejercicio 2005 al $81 \%$ en el 2016. En el resto de sectores la desviación es mucho más pequeña con valores muy próximos a 0 .

Vale destacar que ninguno de los 33 grupos estudiados revela la información completa debido a que nadie describe los factores que configuran el fondo de comercio reconocido, quizá, por ser datos de difícil identificación. Sobre el resto de partidas siempre hay alguna entidad que no informa o que no lo hace de la forma correcta.

Mas del $80 \%$ de los grupos utiliza el valor uso para determinar el valor recuperable del intangible, siendo en la mayoría de los casos un período de 5 años sobre el que se proyecta los flujos de efectivo al emplear una tasa de crecimiento baja y un tipo de descuento que oscila entre el 3 y el $12,6 \%$.

Los gastos reconocidos por deterioro de valor del fondo de comercio son, en términos generales, bajos; y en la mayoría de los casos por importes inferiores al $5 \%$, lo que hubiera supuesto si se le hubiera seguido considerando una vida útil limitada. Es el sector financiero el que engloba un mayor número de grupos que contabilizan deterioros y por mayores importes, en especial en los años de la crisis donde en algunos de ellos el fondo de comercio pierde todo su valor.

De acuerdo con los resultados obtenidos podemos calificar de mejorable la información presentada por los grupos examinados, a pesar de la evolución positiva que han experimentado con el paso de los años. No debemos olvidar las características particulares de la muestra con la que se ha trabajado, así como que la información considerada es requerida obligatoriamente por las NIIF y por lo tanto el nivel de cumplimiento debería de haber sido igual o muy próximo al 100 $\%$. 


\section{Referencias}

Avallone, F. and Quagli, A. (2015). Insight into the variables used to manage the goodwill impairment test under IAS 36. Advances in Accounting, 31(1), 107-114.

Baboukardos, D. and Rimmel, G. (2014). Goodwill under IFRS: Relevance and Disclosures in an Unfavorable Environment. Accounting Forum, 38(1), 1-17.

Ballas, A. and Tzovas, C. (2010). An empirical investigation of Greek firms' compliance to IFRS disclosure requirements. International Journal of Managerial and Financial Accounting, 2(1), 40-62.

Beatty, A. and Weber, J. (2006). Accounting Discretion in Fair Value Estimates: An Examination of SFAS 142 Goodwill Impairments. Journal of Accounting Research, 44(2), 257-288.

Bepari, M., Rahman, S. and Mollik, A. (2014). Firms Compliance with the Disclosure Requirements of IFRS for Goodwill Impairment Testing: Effect of the Global Financial Crisis and Other Firm Characteristics. Journal of Accounting \& Organizational Change, 10(1), 116-149.

Biancone, P. (2012). IFRS: Italian Experience on Impairment Test of Goodwill. International Journal of Advances in Management Science, 1(3), 57-85.

Bloom, M. (2009). Accounting for Goodwill. Abacus, 45(3), 379-389.

Botosan, C. and Plumlee, M. (2002). A reexamination of disclosure level and expected cost of equity capital. Journal of Accounting Research, 40(1), 21-41.

Calvo, J. (2011). El fondo de comercio y la información financiera (tesis doctoral). Universidad Nacional de Educación a Distancia, Madrid, España.

Calvo, J., Corona, E. y Bejarano, V. (2013). Valoración posterior del fondo de comercio: ¿más subjetividad para los estados financieros? Revista Contable, 16, 14-39.
Camodeca, R, Almici, A. and Bernardi, M. (2013). Goodwill impairment testing under IFRS before and after the financial crisis: evidence from the UK large listed companies. Problems and Perspectives in Management, 11(3), 17-23.

Cañibano, L. (2006). Armonización de la normativa contable española con las NIC/NIIF. Revista de la Asociación Española de Contabilidad y Administración de Empresas, 76 (Ext.), 16-20.

Cañibano, L. y Gisbert, A. (2007). El proceso de armonización contable internacional, la estrategia europea y la adaptación de la normativa contable en España. Contaduría, 51, 11-40.

Carlin, T. and Finch, N. (2010). Resisting Compliance with IFRS Goodwill Accounting and Reporting Disclosures: Evidence from Australia. Journal of Accounting \& Organizational Change, 6(2), 260-280.

Carvalho, C., Rodrigues, A. and Ferreira, C. (2016). Goodwill and Mandatory Disclosure Compliance: A Critical Review of the Literature. Australian Accounting Review, 26(4), 376-389.

Chen, V. and Gu, F. (2011). Disclosure about Fair Value Measurement of Goodwill Impairment: Does it Improve Transparency? En 34th Annual Congress EAA, Roma, Italy.

Comiskey, E. and Mulford, C. (2010). Goodwill, Triggering Events and Impairment Accounting. Managerial Finance, 36(9), 746-767.

D’Alauro, G. (2013). The Impact of IAS 36 on Goodwill Disclosure: Evidence of the Write-offs and Performance Effects. Intangible Capital, 9(3), 754-799.

Devalle, A. and Rizzato, F. (2012). The quality of mandatory disclosure: the impairment of goodwill. An empirical analysis of European listed companies. Procedia Economics and Finance, 2, 101-108.

Feteke, S., Matis, D. and Lukacs, J. (2008). Factors Influencing the Extent of Corporate Compliance with IFRS. The Case of Hungarian Listed Companies. Recuperado de http://ssrn. com/abstract $=1295722$. 
Filip, A., Jeanjean, T. and Paugam, L. (2015). Using Real Activities to Avoid Goodwill Impairment Losses: Evidence and Effect on Future Performance. Journal of Business Finance \& Accounting, 42(3), 515-554.

Forbes, T. (2007). SFAS 141: The First 5 Years. Recuperado de http://www.intangiblebusiness. com/Reports/SFAS-141-The-First-5-Years 935. html.

Francis, J.R., Khurana, I. and Pereira, R. (2005). Disclosure incentives and effects on cost of capital around the world. Accounting Review, 80(4), 1125-1162.

Guthrie, J. and Pang, T. (2013). Disclosure of Goodwill Impairment under AASB 136 form 2005-2010. Australian Accounting Review, 23(3), 216-331.

Hail, L. (2003). The relationship between voluntary annual report disclosures and firm characteristics in Switzerland. Die Unternehmung, 57(4), 273-290.

Hartwig, F. (2015). Swedish and Dutch listed companies' compliance with IAS 36 paragraph 134. International Journal of Disclosure and Governance, 12(1), 78-105.

Haswell, S. and Langfield-Smith, I. (2008). Fifty-Seven Serious Defects in Australian IFRS. Australian Accounting Review, 18(1), 46-62.

Iatridis, G. and Senftlechner, D. (2014). An Empirical Investigation of Goodwill in Austria: Evidence on Management Change and Cost of Capital. Australian Accounting Review, 24(2), 171-181.

Izzo, M.F., Luciani, V. and Sartori, E. (2013). Impairment of Goodwill: Level of Compliance and Quality of Disclosure during the Crisis. An Analysis of Italian Listed Companies. International Business Research, 6(11), 94-121.

Kang, T. and Pang, H.Y. (2005). Economic developmentand the value relevance of accounting information - A disclosure transparency perspective. Review of Accounting and Finance, $4(1), 5-31$.
Lazar, L. and Velte, P. (2018). Determinants of mandatory goodwill disclosure: the case of impairment testing in Germany. International Journal of Managerial and Financial Accounting, 10(4), 3013-3030.

Mazzi, F., Liberatore, G. and Tsalavoutas, I. (2016). Insights on CFOs' Perceptions about Impairment Testing Under IAS 36. Journal Accounting in Europe, 13(3), 353-379.

Ott, C. and Guenther, T.W. (2010). Determinants of Purchase Price Allocation Decisions. Recuperado de https://papers.ssrn.com/sol3/papers. cfm?abstract_id=1645124.

Paananen, M. (2008). Fair Value Accounting for Goodwill under IFRS: An Exploratory Study of the Comparability in France, Germany and the United Kingdom. Recuperado de https://ssrn. com/abstract $=1275803$.

Petersen, C. and Plenborg, T. (2010). How Do Firms Implement Impairment Test of Goodwill? Abacus, 46(4), 419-446.

Pietro, P. (2012). IFRS: Italian Experience on Impairment Test of Goodwill. International Journal of Advances in Management Science, 1(3), 57-85.

Ramanna, K. (2008). The Implications of unverifiable fair-value accounting: Evidence from the Political economy of goodwill accounting. Journal of Accounting and Economics, 45(2-3), 253-281.

Ramanna, K. and Watts, R.L. (2012). Evidence on the Use of Unverifiable Estimates in Required Goodwill Impairment. Review of Accounting Studies, 17(4), 749-780.

Shalev, R. (2009). The Information Content of Business Combination Disclosure Level. The Accounting Review, 84(1), 239-270.

Shalev, R., Zhang, I. and Zhang, Y. (2013). CEO Compensation and Fair Value Accounting: Evidence from Purchase Price Allocation. Journal of Accounting Research, 51(4), 819-854.

Tsalavoutas, I. and Dionysiou, D. (2014). Value relevance of IFRS mandatory disclosure 
requirements. Journal of Applied Accounting Research, 15(1), 22-42.

Tua, J. (2005). La reforma del ordenamiento contable: situación actual y algunas reflexiones. En V Jornadas de Trabajo de Contabilidad Financiera, ASEPUC, Madrid, España.

Valero,J.(2017). Análisis de la discrecionalidad en el reconocimiento del deterioro del fondo de comercio: un estudio empírico (tesis doctoral). Universidad Complutense de Madrid, Madrid, España.

Watts, R. (2003). Conservatism in Accounting Part I. Explanations and Implications. Accounting Horizons, 17(3), 207-223.

Wines, G., Dagwell, R. and Windsor, C. (2007). Implications of the IFRS goodwill accounting treatment. Managerial Auditing Journal, 22(9), 862-880. 